\title{
Clear benefits of pulse oximetry in neonates-one small step to improving postnatal cardiac care
}

P:

ulse oximetry, a simple test for blood oxygen saturation, has the potential to detect congenital cardiac abnormalities in newborn babies that might be missed by antenatal ultrasonography and neonatal clinical examination. The PulseOx study is the largest test-accuracy investigation of pulse oximetry conducted in the UK to date. The findings of this study, which could improve postnatal care, are published in the Lancet. "Pulse oximetry offers added value to existing techniques and is likely to increase the number of critical lesions detected prior to [hospital] discharge, if it is introduced as a standard screening procedure", explains Dr Andrew Ewer, from the University of Birmingham, UK, who was one of the study investigators.

Congenital heart defects have been reported to occur in 6-9 per 1,000 live births in developed nations, and represent a major cause of infant mortality in these countries. Pulse oximetry is particularly useful for the detection of malformations that rely on circulation through the ductus arteriosus (duct-dependent lesions), such as hypoplastic left heart syndrome and transposition of the great arteries, by measuring of both preductal and postductal oxygen saturation. Although pulse oximetry is an established technique, and has been previously assessed for the detection of heart defects in newborn babies, most of these earlier studies were underpowered to determine the accuracy of the test. Ewer and colleagues designed the PulseOx study with a sample size large enough to prove both the sensitivity and specificity of pulse oximetry in this setting.

This prospective investigation was conducted at six obstetric units between February 2008 and January 2009. During this period, 20,055 babies ( $>34$ weeks gestation) were screened soon after birth (mean 12.4h). Readings were taken from the infant's right hand (preductal saturation) and either foot (postductal saturation). Babies with a $<95 \%$ oxygen saturation in either limb, or a $2 \%$ difference between limbs, were recorded as having hypoxemia. Echocardiography was performed if clinical examination indicated an abnormality or if oxygen saturation remained below the threshold on a second test. On the basis of echocardiography, cardiac abnormalities were classified as 'critical' (defects causing death or requiring intervention before 28 days of age), 'serious' (defects requiring intervention before 1 year of age), 'significant' (defects persisting longer than 6 months of age and requiring regular monitoring or drug treatment, but not classified as 'critical' or 'serious'), and 'nonsignificant' (defects not clinically appreciable and not persisting beyond 6 months of age). Notably, unlike earlier studies of pulse oximetry in newborns, babies who were identified as having heart defects on mid-gestation ultrasonography were included in the PulseOx study.

Pulse oximetry identified 195 babies $(0.8 \%)$ as having abnormal oxygen saturation, and follow-up echocardiography indicated that $26(13 \%)$ had major heart defects (18 critical and 8 serious). In total, major congenital heart defects were identified by echocardiography or database follow-up in 53 babies $(24$ critical and 29 serious), of whom 19 (36\%) had already been suspected of having a defect on the basis of antenatal ultrasonography. Despite having normal pulse oximetry readings, 27 babies were later diagnosed with a critical or serious congenital heart defect (false negatives). None of these infants died as a result of an undetected defect. The sensitivity of pulse oximetry was $75 \%$ for critical defects and $49 \%$ for all major defects. When babies with suspected cardiac abnormalities on antenatal ultrasonography were excluded, sensitivity was $58 \%$ for critical and $29 \%$ for all major lesions. As the investigators highlight in their paper, "if an antenatal detection [rate] of $50 \%$... is assumed, pulse

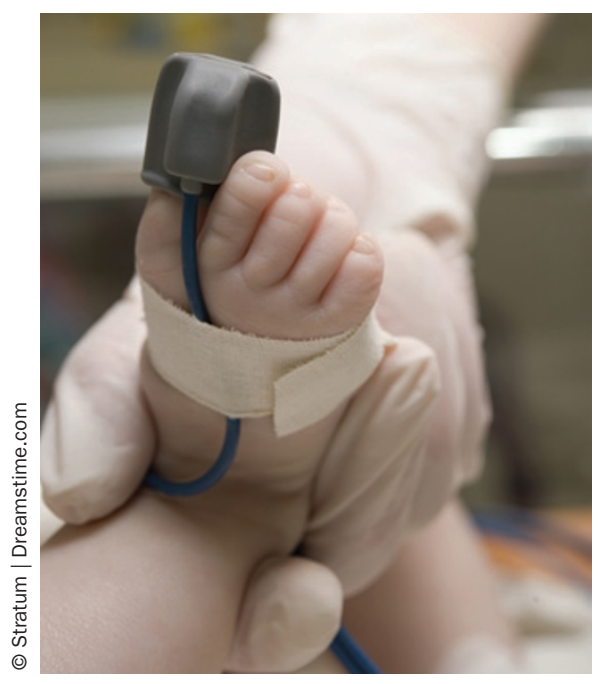

oximetry could detect an additional 35 cases of critical congenital heart defects [per 100,000 births]." Among the 163 babies with a positive pulse oximetry test result but no major or 'significant' congenital heart defect, 40 had other serious conditions (respiratory disorders or infection) requiring treatment. Identification of these conditions was an additional advantage of the test.

Looking to the future, Dr Ewer told us that "we now have sufficient evidence on the accuracy of pulse oximetry screening, and further research in this area is unlikely to yield a different or unexpected finding." However, he goes on to say that "the majority of critical cases missed by pulse oximetry (and by other screening methods) are associated with obstruction of the aortic arch, as these conditions are often not associated with hypoxemia; further investigation of other oximetry techniques, such as perfusion index, may enhance the detection rates for these lesions."

\section{Alexandra King}

Original article Ewer, A. K. et al. Pulse oximetry screening
for congenital heart defects in newborn infants (Pulse0x):
a test accuracy study. Lancet doi:10.1016/
S0140-6736(11)60753-8

